\title{
Design of a Backstepping Tracking Controller for a Class of Linear Systems with Actuator Delay
}

\author{
Yonglong Liao and Fucheng Liao \\ School of Mathematics and Physics, University of Science and Technology Beijing, Beijing 100083, China \\ Correspondence should be addressed to Fucheng Liao; fcliao@ustb.edu.cn
}

Received 29 April 2015; Revised 2 October 2015; Accepted 15 October 2015

Academic Editor: Alfonso Banos

Copyright (C) 2015 Y. Liao and F. Liao. This is an open access article distributed under the Creative Commons Attribution License, which permits unrestricted use, distribution, and reproduction in any medium, provided the original work is properly cited.

\begin{abstract}
This paper presents a method for designing a backstepping tracking controller for a class of continuous-time linear systems with actuator delay subject to a reference signal. The actuator delay can be modeled by a first-order hyperbolic PDE, and then a PDEODE coupled system is obtained. By applying the backstepping transformation to the coupled system, a feedback controller that includes the state of the system, the integral of the input control, and the integral of the tracking error is derived. We show that the closed-loop system is asymptotically stable at the equilibrium point and achieves complete regulation under the stabilizability assumption. The designs in this paper are illustrated with numerical simulations.
\end{abstract}

\section{Introduction}

Over recent decades, many researchers have explored the design of servomechanisms for linear systems with time delay [1-3]. They aimed to design a controller such that the outputs track the reference signals without steady-state errors and the closed-loop system is asymptotically stable at the equilibrium point. They eliminated the influence of time delay on the systems by introducing a predictor that represents the integrator of the time-delay process outputs [4-6]. Or they dealt with the delay in robust control methods for some systems under certain conditions by treating the delay as an uncertain factor [7-9] and then designing the tracking controllers. However, when the plant is unstable, the predictor may fail to achieve closed-loop stability [10], while the robust design method can only solve a portion of specific problems.

Krstic and Smyshlyaev noted that the solution of the firstorder hyperbolic $\operatorname{PDE} u_{t}(s, t)=u_{s}(s, t)$ can be used to replace the actuator delay if the boundary condition is $u(D, t)=$ $U(t)$. Then, the actuator delay systems can be modeled by the PDE-ODE coupled systems. By applying the backstepping transformation, a controller is derived [11]. This kind of controller is equivalent to the classical predictive controller. Moreover, the closed-loop system can be proved to be exponentially stable at the equilibrium point by constructing a Lyapunov-Krasovskii functional. This approach to dealing with delay systems is called backstepping control.

In this way, Bekiaris-Liberis and Krstic [12] constructed an explicit feedback law for systems with simultaneous input and state delay. Krstic and Bresch-Pietri [13] presented an adaptive control design for unstable systems with actuator delay of substantial length and completely unknown value. Other researchers [14-16] have studied systems with timevarying input delay, distributed delay, and pointwise delay, respectively. In their papers, they proved that the designed closed-loop systems are exponentially stable by constructing Lyapunov functionals. Bresch-Pietri et al. [17] made a more intensive study of equilibrium regulation under partial measurements, disturbance rejection, and parameter or delay adaptation. Karafyllis and Krstic [18] provided formulas according to which one can compute estimates of the least upper bound of the magnitude of the delay perturbation. And recently, Lin and Cheng [19] proposed an adaptive block backstepping control scheme for a class of time-delay systems.

The backstepping approach shows a strong superiority in dealing with time-delay systems, but we found almost no systematic results in studying the tracking problem for delay systems with this method. This paper is a new attempt 
to design a tracking controller for a continuous-time linear system with actuator delay, in which we develop a type of servomechanism with PDE style and ODE style state feedback by using the backstepping technique. We formulate the control problem and offer some necessary assumptions in Section 2. A backstepping tracking controller is derived in Section 3 based on the theory of pole placement and the backstepping method. Section 4 shows that the closedloop system is asymptotically stable at the equilibrium point and achieves complete regulation under the stabilizability assumption. In Section 5, we give a practical example and design a backstepping tracking controller; the simulation results demonstrate the effectiveness of the controller. Section 6 is a brief conclusion.

\section{Problem Formulation}

Consider a continuous-time linear system with the actuator delay

$$
\begin{aligned}
& \dot{x}(t)=A x(t)+B U(t-D), \\
& y(t)=C x(t),
\end{aligned}
$$

where $x(t) \in R^{n}$ is the state vector, $U(t-D) \in R$ is the control vector delayed by $D$ units of time, and $y(t) \in R$ is the output vector to be controlled. $A \in R^{n \times n}, B \in R^{n \times 1}$, and $C \in R^{1 \times n}$ are constant matrices.

Let $r(t) \in R$ be the reference signal. The objective of the paper is to develop a feedback controller such that (i) the closed-loop system is asymptotically stable at the equilibrium point and (ii) the output vector $y(t)$ tracks the reference signal $r(t)$; namely,

$$
\lim _{t \rightarrow \infty}(y(t)-r(t))=0
$$

The following assumptions will be needed throughout the paper.

Assumption 1. $(A, B)$ is a controllable pair.

Assumption 2. The reference signal $r(t)$ is a piecewise-continuous function satisfying

$$
\lim _{t \rightarrow \infty} r(t)=r_{0}
$$

where $r_{0}$ is a constant.

Assumption 3. The matrix $\left[\begin{array}{ll}A & B \\ C & 0\end{array}\right]$ has full row rank; that is,

$$
\operatorname{rank}\left[\begin{array}{cc}
A & B \\
C & 0
\end{array}\right]=n+1
$$

\section{Design of Backstepping Tracking Controller}

In this section, we will design a backstepping tracking controller for system (1), so that the closed-loop system is asymptotically stable at the equilibrium point, while the output of the system asymptotically tracks the reference signal.
Define the error vector as

$$
e(t)=y(t)-r(t)
$$

Let $q(t)$ be the integral of the tracking error

$$
q(t)=\int_{0}^{t} e(\tau) \mathrm{d} \tau .
$$

In order to ensure that the system's output tracks the reference signal asymptotically, we need to obtain the integral of the tracking error as part of the state variables. Differentiating both sides of (6) derives

$$
\dot{q}(t)=y(t)-r(t) .
$$

Let $X(t)$ be the $(n+1) \times 1$ augmented vector

$$
X(t)=\left[\begin{array}{l}
x(t) \\
q(t)
\end{array}\right] .
$$

Then, it follows from (1) and (7) that

$$
\dot{X}(t)=\bar{A} X(t)+\bar{B} U(t-D)+\bar{G} r(t),
$$

where $\bar{A}, \bar{B}$, and $\bar{G}$ are $(n+1) \times(n+1),(n+1) \times 1$, and $(n+1) \times 1$ constant matrices defined by

$$
\begin{aligned}
& \bar{A}=\left[\begin{array}{ll}
A & 0 \\
C & 0
\end{array}\right], \\
& \bar{B}=\left[\begin{array}{l}
B \\
0
\end{array}\right], \\
& \bar{G}=\left[\begin{array}{c}
0 \\
-I
\end{array}\right] .
\end{aligned}
$$

Consider the following first-order hyperbolic PDE:

$$
\begin{gathered}
u_{t}(s, t)=u_{s}(s, t), \\
\left.u\right|_{s=D}=U(t), \\
\left.u\right|_{t=0}=\varphi(s),
\end{gathered}
$$

$$
s \in[0, D]
$$

In (11), the delay-free control signal $U(t)$ acts as a boundary condition. $u(s, 0) \triangleq \varphi(s)$ is the initial condition. For convenience, we let the history control over the time interval $[-D, 0]$ be $U(t)=\varphi(D+t), t \in[-D, 0]$. It is well known that the hyperbolic PDE (11) has the following explicit solution:

$$
u(s, t)=U(t+s-D)
$$

Then, the output

$$
u(0, t)=U(t-D)
$$


is the $D$ seconds delayed input. For this reason, the delay system (9) can be modeled by the following PDE-ODE coupled system:

$$
\begin{aligned}
\dot{X}(t) & =\bar{A} X(t)+\bar{B} u(0, t)+\bar{G} r(t), \\
u_{t}(s, t) & =u_{s}(s, t), \\
u(D, t) & =U(t) .
\end{aligned}
$$

The input signal $U(t)$ acts both as a controller and as a boundary condition.

So far, we have converted the tracking problem of an actuator delay system into a regulation problem of a PDEODE coupled system. Next, we will design an appropriate state feedback controller $U(t)$ for system (14) such that the closed-loop system is asymptotically stable at the equilibrium point. We now introduce the backstepping transformation of system (14) and the inverse of this transformation in a theorem form.

Theorem 4. Suppose that $(\bar{A}, \bar{B})$ is controllable. Then,

(i) the backstepping transformation

$$
\begin{aligned}
X(t) & =X(t), \\
v(s, t) & =u(s, t)-\int_{0}^{s} K e^{\bar{A}(s-z)} \bar{B} u(z, t) \mathrm{d} z-K e^{\bar{A} s} X(t)
\end{aligned}
$$

maps system (14) into the following target system:

$$
\begin{aligned}
\dot{X}(t) & =(\bar{A}+\bar{B} K) X(t)+\bar{B} v(0, t)+\bar{G} r(t), \\
v_{t}(s, t) & =v_{s}(s, t)-K e^{\bar{A} s} \bar{G} r(t), \\
v(D, t) & =0,
\end{aligned}
$$

where $K^{T} \in R^{n+1}$ is a stabilizing gain vector such that the matrix $\bar{A}+\bar{B} K$ is Hurwitz.

(ii) The transformation (15) is invertible and the inverse transformation has the following form:

$$
\begin{aligned}
X(t)= & X(t) \\
u(s, t)= & v(s, t)+\int_{0}^{s} K e^{(\bar{A}+\bar{B} K)(s-z)} \bar{B} v(z, t) \mathrm{d} z \\
& +K e^{(\bar{A}+\bar{B} K) s} X(t) .
\end{aligned}
$$

Proof. Let us prove the first part of the theorem. Considering the second equation in (15) with $s=0$, we get

$$
u(0, t)=v(0, t)+K X(t) .
$$

Substituting this expression into the first equation in (14), we get

$$
\dot{X}(t)=(\bar{A}+\bar{B} K) X(t)+\bar{B} v(0, t)+\bar{G} r(t),
$$

that is, the first equation in (16). Let us calculate the time derivatives of the second equation in (15):

$$
\begin{aligned}
v_{t}(s, t)= & u_{t}(s, t)-\int_{0}^{s} K e^{\bar{A}(s-z)} \bar{B} u_{t}(z, t) \mathrm{d} z \\
& -K e^{\bar{A} s} \dot{X}(t) .
\end{aligned}
$$

Using $u_{t}(s, t)=u_{s}(s, t)$, we have

$$
\begin{aligned}
v_{t}(s, t)= & u_{s}(s, t)-\int_{0}^{s} K e^{\bar{A}(s-z)} \bar{B} u_{z}(z, t) \mathrm{d} z \\
& -K e^{\bar{A} s}(\bar{A} X(t)+\bar{B} u(0, t)+\bar{G} r(t)) .
\end{aligned}
$$

After a simple calculation,

$$
\begin{aligned}
v_{t}(s, t)= & u_{s}(s, t)-K \bar{B} u(s, t) \\
& -\int_{0}^{s} K \bar{A} e^{\bar{A}(s-z)} \bar{B} u(z, t) \mathrm{d} z-K e^{\bar{A} s} \bar{A} X(t) \\
& -K e^{\bar{A} s} \bar{G} r(t) .
\end{aligned}
$$

Calculating the spatial derivatives of the second equation in (15) yields

$$
\begin{aligned}
v_{s}(s, t)= & u_{s}(s, t)-K \bar{B} u(s, t) \\
& -\int_{0}^{s} K \bar{A} e^{\bar{A}(s-z)} \bar{B} u(z, t) \mathrm{d} z-K \bar{A} e^{\bar{A} s} X(t) .
\end{aligned}
$$

Subtracting (23) from (22) leads to

$$
v_{t}(s, t)-v_{s}(s, t)=-K e^{\bar{A} s} \bar{G} r(t) .
$$

With $v_{s}(s, t)$ moved to the right side of the equation, we get the second equation in (16). Thus, we prove that the backstepping transformation (15) maps system (14) into the target system (16).

We will offer a straightforward method to prove the second part of this theorem. Note that if we substitute (17) into (15) and get an identity, then we demonstrate that (17) and (15) are inverse.

Substituting (17) into (15), we have

$$
\begin{aligned}
& X(t)=X(t), \\
& v(s, t)=v(s, t)+\int_{0}^{s} K e^{(\bar{A}+\bar{B} K)(s-z)} \bar{B} v(z, t) \mathrm{d} z \\
& +K e^{(\bar{A}+\bar{B} K) s} X(t)-\int_{0}^{s} K e^{\bar{A}(s-z)} \bar{B}(v(z, t) \\
& +\int_{0}^{z} K e^{(\bar{A}+\bar{B} K)(z-h)} \bar{B} v(h, t) \mathrm{d} h \\
& \left.\quad+K e^{(\bar{A}+\bar{B} K) z} X(t)\right) \mathrm{d} z-K e^{\bar{A} s} X(t) .
\end{aligned}
$$


Then, we only need to demonstrate that (26) is an identity. Taking a collection of the similar items in $X$ and $v$, we get

$$
\begin{aligned}
& v(s, t)=v(s, t)+\left(K e^{(\bar{A}+\bar{B} K) s}\right. \\
& \left.-\int_{0}^{s} K e^{\bar{A}(s-z)} \bar{B} K e^{(\bar{A}+\bar{B} K) z} \mathrm{~d} z-K e^{\bar{A} s}\right) X(t) \\
& +\left[\int_{0}^{s} K e^{(\bar{A}+\bar{B} K)(s-z)} \bar{B} v(z, t) \mathrm{d} z\right. \\
& -\int_{0}^{s} K e^{\bar{A}(s-z)} \bar{B} v(z, t) \mathrm{d} z \\
& \left.-\int_{0}^{s} K e^{\bar{A}(s-z)} \bar{B}\left(\int_{0}^{z} K e^{(\bar{A}+\bar{B} K)(z-h)} \bar{B} v(h, t) \mathrm{d} h\right) \mathrm{d} z\right] .
\end{aligned}
$$

For the second part on the right hand of (27), we add $K \int_{0}^{s} e^{\bar{A}(s-z)} \bar{A} e^{(\bar{A}+\bar{B} K) z} \mathrm{~d} z$ to the first integral and then subtract it. By integral calculation, we get

$$
\begin{aligned}
& \left(K e^{(\bar{A}+\bar{B} K) s}-\int_{0}^{s} K e^{\bar{A}(s-z) \bar{B}} K e^{(\bar{A}+\bar{B} K) z} \mathrm{~d} z-K e^{\bar{A} s}\right) \\
& \cdot X(t)=\left(K e^{(\bar{A}+\bar{B} K) s}\right. \\
& -K \int_{0}^{s} e^{\bar{A}(s-z)}(\bar{A}+\bar{B} K) e^{(\bar{A}+\bar{B} K) z} \mathrm{~d} z \\
& \left.+K \int_{0}^{s} e^{\bar{A}(s-z)} \bar{A} e^{(\bar{A}+\bar{B} K) z} \mathrm{~d} z-K e^{\bar{A} s}\right) X(t) \\
& =\left(K e^{(\bar{A}+\bar{B} K) s}-\left.K e^{\bar{A}(s-z)} e^{(\bar{A}+\bar{B} K) z}\right|_{0} ^{s}\right. \\
& -K \int_{0}^{s} \bar{A} e^{\bar{A}(s-z)} e^{(\bar{A}+\bar{B} K) z} \mathrm{~d} z \\
& \left.+K \int_{0}^{s} e^{\bar{A}(s-z)} \bar{A} e^{(\bar{A}+\bar{B} K) z} \mathrm{~d} z-K e^{\bar{A} s}\right) X(t)=0 .
\end{aligned}
$$

And for the third part, we add $\int_{0}^{s} K e^{\bar{A}(s-z)}\left(\int_{0}^{z} \bar{A} e^{(\bar{A}+\bar{B} K)(z-h)} \bar{B} v(h, t) \mathrm{d} h\right) \mathrm{d} z \quad$ to the third integral and then, subtracting it, we have

$$
\begin{gathered}
\int_{0}^{s} K e^{(\bar{A}+\bar{B} K)(s-z)} \bar{B} v(z, t) \mathrm{d} z-\int_{0}^{s} K e^{\bar{A}(s-z)} \bar{B} v(z, t) \mathrm{d} z \\
-\int_{0}^{s} K e^{\bar{A}(s-z)} \bar{B}\left(\int_{0}^{z} K e^{(\bar{A}+\bar{B} K)(z-h)} \bar{B} v(h, t) \mathrm{d} h\right) \mathrm{d} z \\
=K\left[\int_{0}^{s} e^{(\bar{A}+\bar{B} K)(s-z)} v(z, t) \mathrm{d} z-\int_{0}^{s} e^{\bar{A}(s-z)} v(z, t) \mathrm{d} z\right. \\
-\int_{0}^{s} e^{\bar{A}(s-z)}\left(\int_{0}^{z}(\bar{A}+\bar{B} K) e^{(\bar{A}+\bar{B} K)(z-h)} v(h, t) \mathrm{d} h\right) \mathrm{d} z \\
\left.+\int_{0}^{s} e^{\bar{A}(s-z)}\left(\int_{0}^{z} \bar{A} e^{(\bar{A}+\bar{B} K)(z-h)} v(h, t) \mathrm{d} h\right) \mathrm{d} z\right] \bar{B} .
\end{gathered}
$$

Then, exchanging integral order and calculating it, we get

$$
\begin{aligned}
& \int_{0}^{s} K e^{(\bar{A}+\bar{B} K)(s-z)} \bar{B} v(z, t) \mathrm{d} z-\int_{0}^{s} K e^{\bar{A}(s-z)} \bar{B} v(z, t) \mathrm{d} z \\
& -\int_{0}^{s} K e^{\bar{A}(s-z)} \bar{B}\left(\int_{0}^{z} K e^{(\bar{A}+\bar{B} K)(z-h)} \bar{B} v(h, t) \mathrm{d} h\right) \mathrm{d} z \\
& =K\left[\int_{0}^{s} e^{(\bar{A}+\bar{B} K)(s-z)} v(z, t) \mathrm{d} z-\int_{0}^{s} e^{\bar{A}(s-z)} v(z, t) \mathrm{d} z\right. \\
& -\int_{0}^{s}\left(\int_{h}^{s} e^{\bar{A}(s-z)}(\bar{A}+\bar{B} K) e^{(\bar{A}+\bar{B} K)(z-h)} \mathrm{d} z\right) \\
& \cdot v(h, t) \mathrm{d} h+\int_{0}^{s}\left(\int_{h}^{s} e^{\bar{A}(s-z)} \bar{A} e^{(\bar{A}+\bar{B} K)(z-h)} \mathrm{d} z\right) \\
& \cdot v(h, t) \mathrm{d} h] \bar{B}=K\left[\int_{0}^{s} e^{(\bar{A}+\bar{B} K)(s-z)} v(z, t) \mathrm{d} z\right. \\
& -\int_{0}^{s} e^{\bar{A}(s-z)} v(z, t) \mathrm{d} z-\int_{0}^{s}\left(\left.e^{\bar{A}(s-z)} e^{(\bar{A}+\bar{B} K)(z-h)}\right|_{h} ^{s}\right. \\
& \left.+\int_{h}^{s} \bar{A} e^{\bar{A}(s-z)} e^{(\bar{A}+\bar{B} K)(z-h)} \mathrm{d} z\right) v(h, t) \mathrm{d} h \\
& \left.+\int_{0}^{s}\left(\int_{h}^{s} e^{\bar{A}(s-z)} \bar{A} e^{(\bar{A}+\bar{B} K)(z-h)} \mathrm{d} z\right) v(h, t) \mathrm{d} h\right] \bar{B}=0 .
\end{aligned}
$$

Thus, (26) is an identity

$$
v(s, t)=v(s, t)
$$

which illustrates that (15) and (17) are inverse transforms with each other. The proof is complete.

In the proof of Theorem 4, we have not mentioned the third equation in (16). In fact, the boundary condition $v(D, t)$ of (16) depends not only on the transformation of (15), but also on the boundary input $u(D, t)$ for (14). In order to get the desired results, we let

$$
v(D, t)=0 .
$$

Then, the boundary input for (14) should be

$$
u(D, t)=\int_{0}^{D} K e^{\bar{A}(D-z)} \bar{B} u(z, t) \mathrm{d} z+K e^{\bar{A} D} X(t)
$$

Equation (33) is the feedback controller for (14). Noting that $X(t)=\left[x(t)^{T} q(t)^{T}\right]^{T}$, we give the backstepping tracking controller $U(t)$ for (1) by the following theorem.

Theorem 5. Suppose that $(\bar{A}, \bar{B})$ is controllable and the reference signal satisfies Assumption 2. Then, the backstepping tracking controller $U(t)$ is given by

$$
\begin{aligned}
U(t)= & \int_{0}^{D} K e^{\bar{A}(D-z)} \bar{B} u(z, t) \mathrm{d} z+K_{x} x(t) \\
& +K_{e} \int_{0}^{t} e(\tau) \mathrm{d} \tau
\end{aligned}
$$


where $K \in R^{1 \times(n+1)}$ is a stabilizing gain vector such that the matrix $\bar{A}+\bar{B} K$ is Hurwitz. $K_{x} \in R^{1 \times n}$ and $K_{e} \in R^{1 \times 1}$ are the feedback gains defined by

$$
K e^{\bar{A} D}=\left[\begin{array}{ll}
K_{x} & K_{e}
\end{array}\right] .
$$

Remark 6. We can find that the backstepping tracking controller consists of three parts. The first is the feedback in partial differential form, and its essence is the feedback of the input delay integral, which is used to offset the lag input's effect on the system. The second part is the feedback of the present state. And the third part is the integral action on the tracking error: it guarantees that the output tracks the reference signal asymptotically.

Remark 7. Specifically, if $D=0$, then (34) is

$$
U(t)=K_{x} x(t)+K_{e} \int_{0}^{t} e(\tau) \mathrm{d} \tau
$$

which consists of the feedback of the state and integral of error but does not consist of the PDE section. This indicates that the effect of introducing PDE is to eliminate the input delay's impact formally. On the other hand, $K e^{\bar{A} D}=K$ when $D=0$, and then $\bar{A}+\bar{B} K$ is stable. This is consistent with the results of general control theory and is equivalent to the controller design with the pole placement method.

In fact, with the backstepping coordinate transformation of (15), the closed-loop system constituted by (14) and (33) is (16). If we can prove that the closed-loop system (16) is asymptotically stable at the equilibrium point, then we have demonstrated that (34) is the exact controller we want to design.

\section{Stability of the Closed-Loop System}

In this section, we will consider the stability and tracking property of the closed-loop system. Two lemmas are needed.

Lemma 8 (see [20]). The pair $(\bar{A}, \bar{B})$ is controllable if and only if $(A, B)$ is controllable and the matrix $\Phi=\left[\begin{array}{cc}A & B \\ C & 0\end{array}\right]$ has full row rank.

Lemma 9 (see [21]). Consider the linear continuous-time system

$$
\begin{aligned}
& \dot{x}(t)=A x(t)+f(t), \\
& x(0)=x_{0} \in R^{n},
\end{aligned}
$$

where $A \in R^{n \times n}$ is Hurwitz and $f(\cdot)$ is a bounded measurable function on $[0, \infty)$. And $\lim _{t \rightarrow \infty} f(t)=f^{*} \in R^{n}$. Then for any $x_{0} \in R^{n}$ the state vector $x(t)$ satisfies

$$
\lim _{t \rightarrow \infty} x(t)=-A^{-1} f^{*} .
$$

Now we present the stability theorem of the closed-loop system (16).
Theorem 10. Suppose that $(A, B)$ is controllable and the matrix $\Phi=\left[\begin{array}{cc}A & B \\ C & 0\end{array}\right]$ has full row rank, and the reference signal is asymptotically stable. Then, the closed-loop system (16) is asymptotically stable at the equilibrium point. And the output of system (1) asymptotically tracks the reference signal under the controller (34); namely,

$$
\lim _{t \rightarrow \infty} e(t)=0 .
$$

Proof. We first show that the closed-loop system is asymptotically stable at the equilibrium point. Under the conditions Assumptions 1 and 3, it follows from Lemma 8 that $(\bar{A}, \bar{B})$ is controllable and there exists a matrix $K^{T} \in R^{n+1}$ such that $\bar{A}+\bar{B} K$ is Hurwitz. Let $\left[\begin{array}{c}X^{*} \\ v(s)^{*}\end{array}\right]$ be the equilibrium point of (16): it follows that

$$
\begin{aligned}
(\bar{A}+\bar{B} K) X^{*}+\bar{B} v(0)^{*}+\bar{G} r_{0} & =0, \\
\frac{d v(s)^{*}}{d s}-K e^{\overline{A s}} \bar{G} r_{0} & =0, \\
v(D)^{*} & =0 .
\end{aligned}
$$

Solving (40), we get

$$
\begin{aligned}
X^{*} & =-(\bar{A}+\bar{B} K)^{-1}\left(\bar{G} r_{0}-\bar{B} \int_{0}^{D} K e^{\bar{A} \tau} \bar{G} r_{0} \mathrm{~d} \tau\right), \\
v(s)^{*} & =-\int_{s}^{D} K e^{\bar{A} \tau} \bar{G} r_{0} \mathrm{~d} \tau .
\end{aligned}
$$

Letting

$$
\begin{aligned}
\widetilde{X}(t) & =X(t)-X^{*}, \\
\widetilde{v}(s, t) & =v(s, t)-v(s)^{*}
\end{aligned}
$$

we transform (16) into

$$
\begin{aligned}
\dot{\tilde{X}}(t) & =(\bar{A}+\bar{B} K) \widetilde{X}(t)+\bar{B} \widetilde{v}(0, t)+\bar{G}\left(r(t)-r_{0}\right), \\
\widetilde{v}_{t}(s, t) & =\widetilde{v}_{s}(s, t)-K e^{\bar{A} s} \bar{G}\left(r(t)-r_{0}\right), \\
\widetilde{v}(D, t) & =0 .
\end{aligned}
$$

Now, we give a detailed demonstration of the asymptotic stability of (43) through direct calculation.

We consider the partial differential equation in (43) first. According to $u(s, 0)=\varphi(s)$ and $\varphi(D+t)=U(t)(t \in[-D, 0])$ in Section 3, we have $u(s, 0)=U(s-D)(s \in[0, D])$. That is, the initial condition of the partial differential equation $u(s, 0)$ is determined by the initial input $U(t)$ on $t \in[-D, 0]$. Hence, $\widetilde{v}(s, 0)$ is determined by $r_{0}$ and the initial input $U(t)$. For briefness in calculation, we let $\widetilde{v}(s, 0)=0$ by giving a suitable initial input. It should be pointed out that the proof is also correct when $\widetilde{v}(s, 0) \neq 0$.

Thus, the partial differential equation in (43) with initial and boundary condition is described as follows:

$$
\begin{aligned}
\widetilde{v}_{t}(s, t) & =\widetilde{v}_{s}(s, t)-K e^{\bar{A} s} \bar{G}\left(r(t)-r_{0}\right), \\
\widetilde{v}(D, t) & =0, \\
\widetilde{v}(s, 0) & =0 .
\end{aligned}
$$


Solving (44) and letting $r(t)=r_{0}(t<0)$, we obtain

$$
\widetilde{v}(s, t)=\int_{D}^{s} K e^{\bar{A} \tau} \bar{G}\left(r(t+s-\tau)-r_{0}\right) \mathrm{d} \tau .
$$

Taking the norm on both sides of (45), we have

$$
\begin{aligned}
& \|\widetilde{v}(s, t)\| \\
& \quad \leq \max _{\tau \in[s, D]}\left\|K e^{\bar{A} \tau} \bar{G}\right\| \max _{\tau \in[s, D]}\left\|r(t+s-\tau)-r_{0}\right\| D .
\end{aligned}
$$

According to Assumption 2, we have $\lim _{t \rightarrow \infty}\left(r(t)-r_{0}\right)=0$. Thus, we have

$$
\lim _{t \rightarrow \infty}\|\widetilde{v}(s, t)\|=0
$$

On the other hand, according to (45) we have

$$
\widetilde{v}(0, t)=\int_{D}^{0} K e^{\bar{A} \tau} \bar{G}\left(r(t-\tau)-r_{0}\right) \mathrm{d} \tau .
$$

Substituting (48) into the first equation of (43), we get

$$
\begin{aligned}
\dot{\widetilde{X}}(t)= & (\bar{A}+\bar{B} K) \widetilde{X}(t) \\
& +\bar{B} \int_{D}^{0} K e^{\bar{A} \tau} \bar{G}\left(r(t-\tau)-r_{0}\right) \mathrm{d} \tau \\
& +\bar{G}\left(r(t)-r_{0}\right) .
\end{aligned}
$$

Let

$$
\begin{aligned}
f(t)= & \bar{B} \int_{D}^{0} K e^{\bar{A} \tau} \bar{G}\left(r(t-\tau)-r_{0}\right) \mathrm{d} \tau \\
& +\bar{G}\left(r(t)-r_{0}\right) .
\end{aligned}
$$

Equation (49) is written as

$$
\dot{\widetilde{X}}(t)=(\bar{A}+\bar{B} K) \widetilde{X}(t)+f(t) .
$$

For (50), the following inequality holds:

$$
\begin{aligned}
\|f(t)\| \leq & \|\bar{B} K\|\|\bar{G}\| \max _{\tau \in[0, D]}\left\|e^{\bar{A} \tau}\right\|\left|r(t-\tau)-r_{0}\right| D \\
& +\|\bar{G}\|\left|r(t)-r_{0}\right| .
\end{aligned}
$$

Combining $\lim _{t \rightarrow \infty}\left(r(t)-r_{0}\right)=0$, we have

$$
\lim _{t \rightarrow \infty} f(t)=0 \text {. }
$$

Using Lemma 9, we obtain

$$
\lim _{t \rightarrow \infty} \widetilde{X}(t)=-(\bar{A}+\bar{B} K)^{-1} \lim _{t \rightarrow \infty} f(t)=0 .
$$

Combining (47) and (54), we have

$$
\lim _{t \rightarrow \infty}(\|\widetilde{X}(t)\|+\|\widetilde{v}(s, t)\|)=0
$$

which indicates that (43) is asymptotically stable.
Obviously, the target system (16) is also asymptotically stable at the equilibrium point because (42) is just a coordinate moving in parallel with (16). And the equilibrium point is

$$
\left[\begin{array}{c}
X(t) \\
v(s, t)
\end{array}\right]=\left[\begin{array}{c}
X^{*} \\
v(s)^{*}
\end{array}\right] .
$$

Hence, the statement in Section 1 is proved.

From the discussions above, we have

$$
\begin{aligned}
& \lim _{t \rightarrow \infty} X(t)=X^{*}, \\
& \lim _{t \rightarrow \infty} U(t)=U^{*},
\end{aligned}
$$

where $X^{*}, U^{*}$ are constant vectors and satisfy

$$
\bar{A} X^{*}+\bar{B} U^{*}+\bar{G} r_{0}=0 .
$$

Denoting $X^{*}=\left[\begin{array}{c}x^{*} \\ q^{*}\end{array}\right],(58)$ can also be written as

$$
\left[\begin{array}{ll}
A & 0 \\
C & 0
\end{array}\right]\left[\begin{array}{l}
x^{*} \\
q^{*}
\end{array}\right]+\left[\begin{array}{l}
B \\
0
\end{array}\right] U^{*}+\left[\begin{array}{c}
0 \\
-I
\end{array}\right] r_{0}=0
$$

We have

$$
C x^{*}-r_{0}=0
$$

that is,

$$
\lim _{t \rightarrow \infty}(C x(t)-r(t))=0 .
$$

Namely,

$$
\lim _{t \rightarrow \infty} e(t)=0 .
$$

Equation (62) suggests the system's output asymptotically tracks the reference signal. Thus, the proof of Theorem 10 is complete.

Summarizing the results above, we give the following tracking controller theorem.

Theorem 11. Suppose that Assumptions 1 to 3 are satisfied; then, there exists $K$ such that $\bar{A}+\bar{B} K$ is stable, and the closedloop system of (1) is

$$
\dot{x}(t)=A x(t)+B u(0, t) .
$$

The control input $U(t-D)=u(0, t)$ is determined by the following PDE-ODE coupled system:

$$
\begin{aligned}
& u(s, t)=v(s, t)+\int_{0}^{s} K e^{(\bar{A}+\bar{B} K)(s-z)} \bar{B} v(z, t) \mathrm{d} z \\
& +K e^{(\bar{A}+\bar{B} K) s}\left[\begin{array}{c}
x(t) \\
\int_{0}^{t}[C x(\tau)-r(\tau)] \mathrm{d} \tau
\end{array}\right], \\
& v_{t}(s, t)=v_{s}(s, t)-K e^{\bar{A} s} \bar{G} r(t), \\
& v(D, t)=0 \text {. }
\end{aligned}
$$




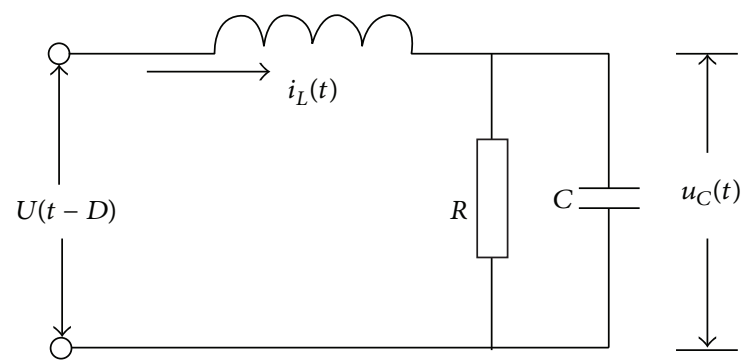

FIGURE 1: An RLC actuator delay network.

Theorem 11 illustrates that $v(s, t)$ can be obtained through solving the PDE

$$
\begin{aligned}
& v_{t}(s, t)=v_{s}(s, t)-K e^{\bar{A} s} \bar{G} r(t), \\
& v(D, t)=0 .
\end{aligned}
$$

Then, $u(s, t)$ is obtained from (64), and finally we get the control input $U(t-D)=u(0, t)$.

\section{A Simulation Example}

We will give the actuator delay RLC network [22] described in Figure 1 to illustrate the effectiveness of the controller.

In Figure $1, i_{L}$ is the current through the inductor $L, u_{C}$ is the voltage across the capacitor $C$ and is also the output of the system, $U(t-D)$ is the input voltage which has a time delay to the network, and $R=1 / 3 \Omega, C=1 \mathrm{~F}, L=0.5 \mathrm{H}$, and $D=0.5 \mathrm{~s}$. Now, we make the definition $x_{1}(t)=i_{L}(t)$ and $x_{2}(t)=u_{C}(t)$; applying Kirchhoff's current and voltage laws to the RLC network, we get the following mathematical model:

$$
\dot{x}(t)=A x(t)+B U(t-0.5)
$$

where $x(t)=\left[\begin{array}{l}x_{1}(t) \\ x_{2}(t)\end{array}\right], A=\left[\begin{array}{ll}0 & -2 \\ 1 & -3\end{array}\right]$, and $B=\left[\begin{array}{l}2 \\ 0\end{array}\right]$. The output equation is given by

$$
y(t)=C x(t)
$$

where $C=\left[\begin{array}{ll}0 & 1\end{array}\right]$. Let $x(0)=\left[\begin{array}{l}0 \\ 0\end{array}\right]$ and $U(t)=0,(-0.5 \leq t \leq$ $0)$.

We give the reference output of $u_{C}$ as the following four types.

Step Signal

$$
r(t)= \begin{cases}0, & t<15 \\ 1, & t \geq 15\end{cases}
$$

Periodic Signal

$$
r(t)= \begin{cases}\sin \left(\frac{\pi}{5}(t-3)\right), & 3<t \leq 53 \\ 0, & \text { other. }\end{cases}
$$

Ramp Signal

$$
r(t)= \begin{cases}0, & t \leq 10, \\ \frac{1}{20}(t-10), & 10<t \leq 30, \\ 1, & t>30 .\end{cases}
$$

Fading Signal

$$
r(t)= \begin{cases}0, & t \leq 5, \\ \frac{1}{t} \sin \left(\frac{\pi}{5}(t-5)\right), & t>5 .\end{cases}
$$

The control system can be described as

$$
\begin{aligned}
\dot{x}(t) & =A x(t)+B U(t-0.5), \\
y(t) & =C x(t), \\
e(t) & =y(t)-r(t), \\
u_{t}(s, t) & =u_{s}(s, t), \\
u(0.5, t) & =U(t) .
\end{aligned}
$$

And the control input $U(t-0.5)=u(0, t)$ is given by the following PDE-ODE coupled system:

$$
\begin{aligned}
& u(s, t)=v(s, t)+\int_{0}^{s} K e^{(\bar{A}+\bar{B} K)(s-z)} \bar{B} v(z, t) \mathrm{d} z \\
& +K e^{(\bar{A}+\bar{B} K) s}\left[\begin{array}{c}
x(t) \\
\int_{0}^{t}[C x(\tau)-r(\tau)] \mathrm{d} \tau
\end{array}\right], \\
& v_{t}(s, t)=v_{s}(s, t)-K e^{\bar{A} s} \bar{G} r(t), \\
& v(D, t)=0 \text {, }
\end{aligned}
$$

where $\bar{A}=\left[\begin{array}{ccc}0 & -2 & 0 \\ 1 & -3 & 0 \\ 0 & 1 & 0\end{array}\right], \bar{B}=\left[\begin{array}{l}2 \\ 0 \\ 0\end{array}\right]$, and $\bar{G}=\left[\begin{array}{c}0 \\ 0 \\ -1\end{array}\right]$. Prescribing the poles $\left[\begin{array}{lll}-9 & -10 & -8\end{array}\right]$ to the nominal system (14) results in

$$
K=\left[\begin{array}{lll}
-12 & -84 & -360
\end{array}\right]
$$

and hence we have

$$
K_{x}=K e^{\bar{A} D}\left[\begin{array}{ll}
1 & 0 \\
0 & 1 \\
0 & 0
\end{array}\right],
$$

$$
K_{e}=K e^{\bar{A} D}\left[\begin{array}{l}
0 \\
0 \\
1
\end{array}\right] .
$$

The system response under the backstepping tracking controller is depicted in Figures 2, 3, 4, and 5. It can be observed that the backstepping tracking controller can indeed guarantee that the output tracks the reference signals asymptotically. That is to say, the design method of the controller in this paper is effective. 


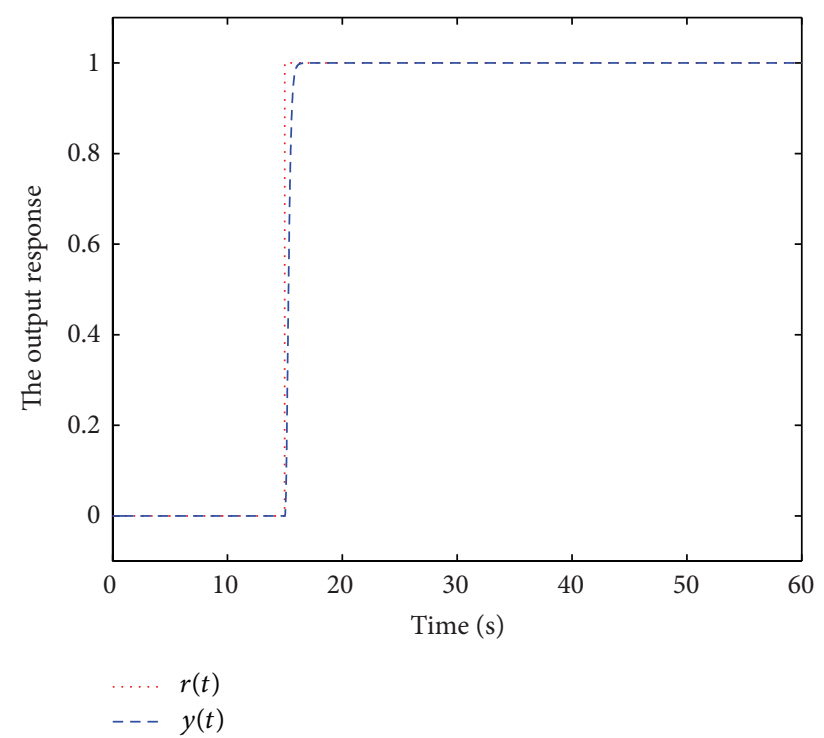

Figure 2: Closed-loop responses of step signal (68).

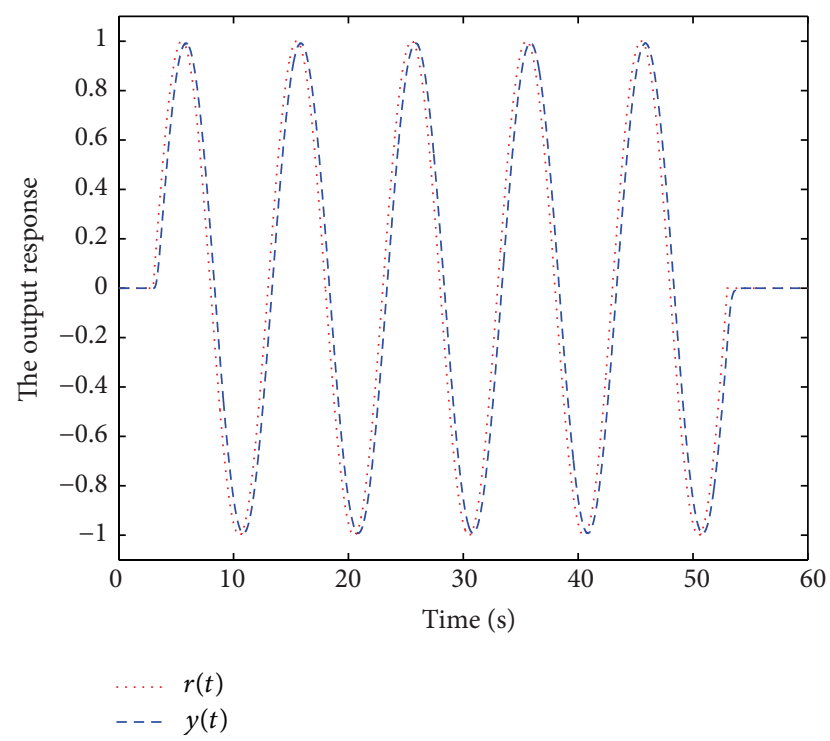

FIGURE 3: Closed-loop responses of periodic signal (69).

\section{Conclusion}

In this paper, we have proposed a backstepping tracking control scheme for a class of linear systems with actuator delay. By constructing a PDE-ODE coupled system based on the augmented system constructed, we converted the tracking problem into a regulation one. The proposed control scheme can effectively track the reference signal and guarantee the asymptotic stability of the closed-loop system. The numerical simulations show the efficiency of the backstepping tracking controller.

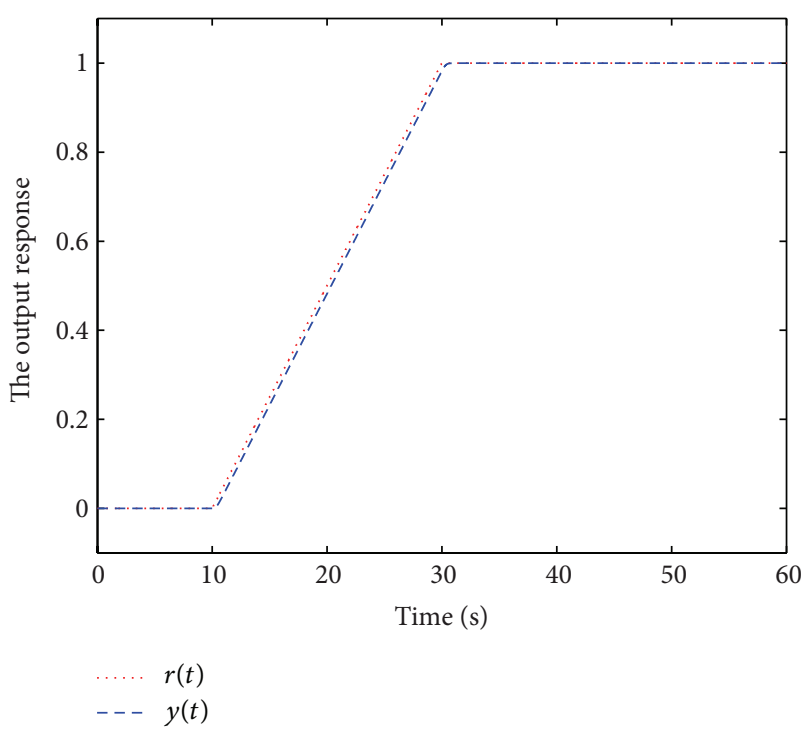

FIGURE 4: Closed-loop responses of ramp signal (70).

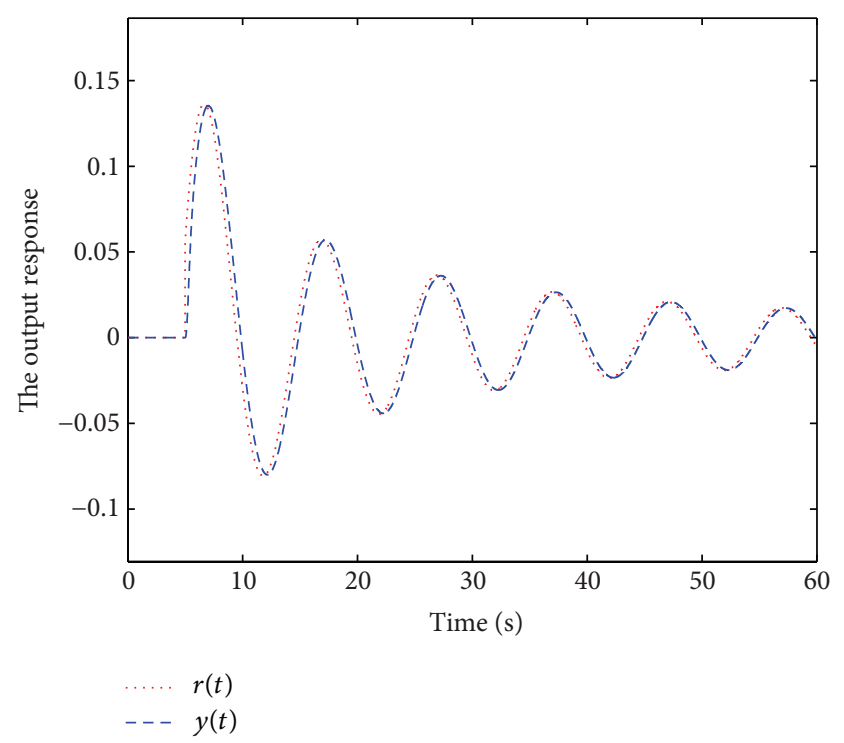

FIGURE 5: Closed-loop responses of fading signal (71).

\section{Appendix}

The backstepping transformation (15) can be obtained by this method. Let the transformation have the following form:

$$
\begin{aligned}
X(t)= & X(t) \\
v(s, t)= & u(s, t)-\int_{0}^{s} k(s, z) u(z, t) \mathrm{d} z \\
& -\gamma(s)^{T} X(t)
\end{aligned}
$$


where $k(s, z) \in R$ and $\gamma(s) \in R^{n+1}$ are kernels to be determined and $K^{T} \in R^{n+1}$ satisfying $\bar{A}+\bar{B} K$ is Hurwitz. This converts plant (14) into the following target system:

$$
\begin{aligned}
\dot{X}(t) & =(\bar{A}+\bar{B} K) X(t)+\bar{B} v(0, t)+\bar{G} r(t), \\
v_{t}(s, t) & =v_{s}(s, t)-\gamma(s)^{T} \bar{G} r(t), \\
v(D, t) & =0 .
\end{aligned}
$$

Calculating the time derivatives of the second equation in (A.1),

$$
\begin{aligned}
v_{t}(s, t)= & u_{s}(s, t)-k(s, s) u(s, t)+k(s, 0) u(0, t) \\
& +\int_{0}^{s} k_{z}(s, z) u(z, t) \mathrm{d} z-\gamma(s)^{T} \bar{A} X(t) \\
& -\gamma(s)^{T} \bar{B} u(0, t)-\gamma(s)^{T} \bar{G} r(t),
\end{aligned}
$$

and calculating the spatial derivatives of the second equation in (A.1),

$$
\begin{aligned}
v_{s}(s, t)= & u_{s}(s, t)-k(s, s) u(s, t) \\
& -\int_{0}^{s} k_{s}(s, z) u(z, t) \mathrm{d} z-\gamma^{\prime}(s)^{T} X(t),
\end{aligned}
$$

by the target system (A.2) we get

$$
\begin{aligned}
& v_{t}(s, t)-v_{s}(s, t)+\gamma(s)^{T} \bar{G} r(t) \\
& =\int_{0}^{s}\left(k_{s}(s, z)+k_{z}(s, z)\right) u(z, t) \mathrm{d} z \\
& \quad+\left(k(s, 0)-\gamma(s)^{T} \bar{B}\right) u(0, t) \\
& \quad+\left(\gamma^{\prime}(s)^{T}-\gamma(s)^{T} \bar{A}\right) X(t)=0 .
\end{aligned}
$$

This equation should be valid for all $u(s, t), u(0, t)$, and $X(t)$, so we have

$$
\begin{aligned}
k_{s}(s, z)+k_{z}(s, z) & =0, \\
k(s, 0)-\gamma(s)^{T} \bar{B} & =0, \\
\gamma^{\prime}(s)^{T} & =\gamma(s)^{T} \bar{A} .
\end{aligned}
$$

On the other hand, let $s=0$ in the second equation in (A.1), which gives

$$
v(0, t)=u(0, t)-\gamma(0)^{T} X(t) .
$$

Substituting (A.7) into the first equation in (14) and (A.2), we have

$$
\left(K-\gamma^{T}(0)\right) X(t)=0
$$

So

$$
\gamma^{T}(0)=K \text {. }
$$

The kernels $k(s, z)$ and $\gamma(s)$ should satisfy

$$
\begin{aligned}
k_{s}(s, z)+k_{z}(s, z) & =0, \\
k(s, 0)-\gamma(s)^{T} \bar{B} & =0, \\
\gamma^{\prime}(s)^{T} & =\gamma(s)^{T} \bar{A}, \\
\gamma^{T}(0) & =K .
\end{aligned}
$$

We can easily obtain by (A.11) that $\gamma(s)^{T}=K e^{\bar{A} s}$. Substituting this expression into (A.10), we get $k(s, z)=K e^{\bar{A}(s-z)} \bar{B}$. Thus, we get the transformation (A.1):

$$
\begin{aligned}
X(t)= & X(t), \\
v(s, t)= & u(s, t)-\int_{0}^{s} K e^{\bar{A}(s-z)} \bar{B} u(z, t) \mathrm{d} z \\
& -K e^{\bar{A} s} X(t) .
\end{aligned}
$$

And that is (15).

\section{Conflict of Interests}

The authors declare that there is no conflict of interests regarding the publication of this paper.

\section{Acknowledgments}

This work was supported by the National Natural Science Foundation of China (no. 61174209) and the Oriented Award Foundation for Science and Technological Innovation, Inner Mongolia Autonomous Region, China (2012).

\section{References}

[1] A. T. Bahill, "A simple adaptive Smith-predictor for controlling time-delay systems: a tutorial," IEEE Control Systems Magazine, vol. 3, no. 2, pp. 16-22, 1983.

[2] J. E. Normey-Rico and E. F. Camacho, Control of Dead-Time Processes, Springer, London, UK, 2007.

[3] S. Oucheriah, "Robust tracking and model following of uncertain dynamic delay systems by memoryless linear controllers," IEEE Transactions on Automatic Control, vol. 44, no. 7, pp. 14731477, 1999.

[4] Y. Fiagbedzi and A. E. Pearson, "Feedback stabilization of linear autonomous time lag systems," IEEE Transactions on Automatic Control, vol. 31, no. 9, pp. 847-855, 1986.

[5] S. Majhi and D. P. Atherton, "Obtaining controller parameters for a new Smith predictor using autotuning," Automatica, vol. 36, no. 11, pp. 1651-1658, 2000.

[6] M. R. Stojić, M. S. Matijević, and L. S. Draganović, "A robust Smith predictor modified by internal models for integrating process with dead time," IEEE Transactions on Automatic Control, vol. 46, no. 8, pp. 1293-1298, 2001.

[7] P. H. Chang and J. W. Lee, "A model reference observer for timedelay control and its application to robot trajectory control," IEEE Transactions on Control Systems Technology, vol. 4, no. 1, pp. 2-10, 1996. 
[8] H. Trinh and M. Aldeen, "Output tracking for linear uncertain time-delay systems," IEE Proceedings-Control Theory and Applications, vol. 143, pp. 481-488, 1996.

[9] H. Wu, "Adaptive robust tracking and model following of uncertain dynamical systems with multiple time delays," IEEE Transactions on Automatic Control, vol. 49, no. 4, pp. 611-616, 2004.

[10] M. Krstic, "Compensation of infinite-dimensional actuator and sensor dynamics: nonlinear and delay-adaptive systems," IEEE Control Systems Magazine, vol. 30, no. 1, pp. 22-41, 2010.

[11] M. Krstic and A. Smyshlyaev, "Backstepping boundary control for first-order hyperbolic PDEs and application to systems with actuator and sensor delays," Systems \& Control Letters, vol. 57, no. 9, pp. 750-758, 2008.

[12] N. Bekiaris-Liberis and M. Krstic, "On stabilizing strictfeedback linear systems with delayed integrators," in Proceedings of the 17th Mediterranean Conference on Control and Automation (MED '09), pp. 163-168, Thessaloniki, Greece, June 2009.

[13] M. Krstic and D. Bresch-Pietri, "Delay-adaptive full-state predictor feedback for systems with unknown long actuator delay," in Proceedings of the American Control Conference (ACC '09), pp. 4500-4505, IEEE, St. Louis, Mo, USA, June 2009.

[14] M. Krstic, "Lyapunov stability of linear predictor feedback for time-varying input delay," IEEE Transactions on Automatic Control, vol. 55, no. 2, pp. 554-559, 2010.

[15] N. Bekiaris-Liberis and M. Krstic, "Lyapunov stability of linear predictor feedback for distributed input delays," IEEE Transactions on Automatic Control, vol. 56, no. 3, pp. 655-660, 2011.

[16] F. Mazenc, S.-I. Niculescu, and M. Krstic, "Lyapunov-krasovskii functionals and application to input delay compensation for linear time-invariant systems," Automatica, vol. 48, no. 7, pp. 1317-1323, 2012.

[17] D. Bresch-Pietri, J. Chauvin, and N. Petit, "Adaptive control scheme for uncertain time-delay systems," Automatica, vol. 48, no. 8, pp. 1536-1552, 2012.

[18] I. Karafyllis and M. Krstic, "Delay-robustness of linear predictor feedback without restriction on delay rate," Automatica, vol. 49, no. 6, pp. 1761-1767, 2013.

[19] Y.-S. Lin and C.-C. Cheng, "Design of block backstepping controllers for a class of perturbed multiple inputs and statedelayed systems in semi-strict-feedback form," International Journal of Systems Science, 2014.

[20] T. Katayama and T. Hirono, "Design of an optimal servomechanism with preview action and its dual problem," International Journal of Control, vol. 45, no. 2, pp. 407-420, 1987.

[21] W. Chen, "On convergent properties for states of nonhomogeneous linear systems," Journal of Tianjin University of Light Industry, vol. 18, pp. 1-3, 2003.

[22] Y. Jing, Y. Zhou, and N. Jiang, Modern Control Theory, Metallurgical Industry Press, 2006. 


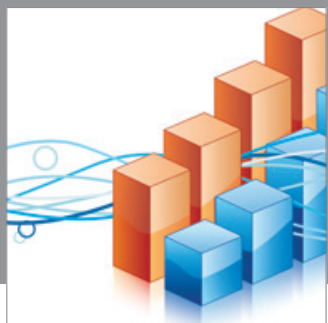

Advances in

Operations Research

mansans

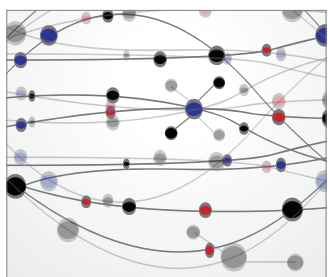

The Scientific World Journal
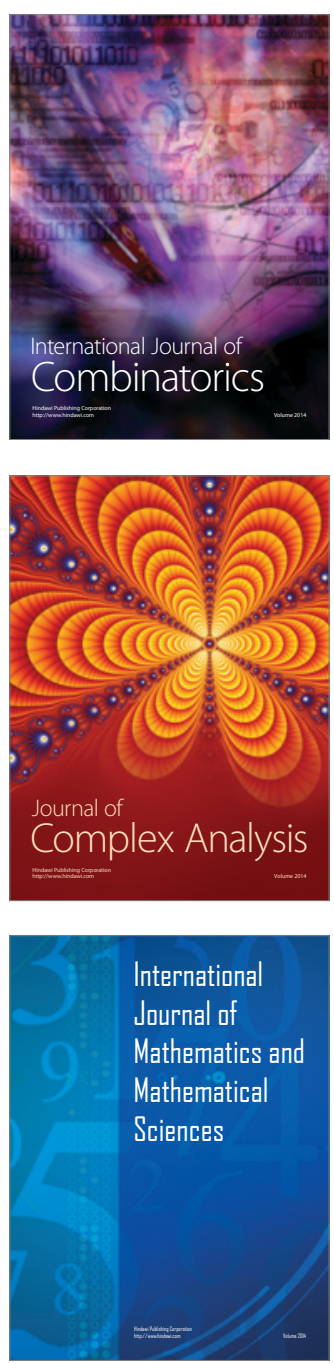
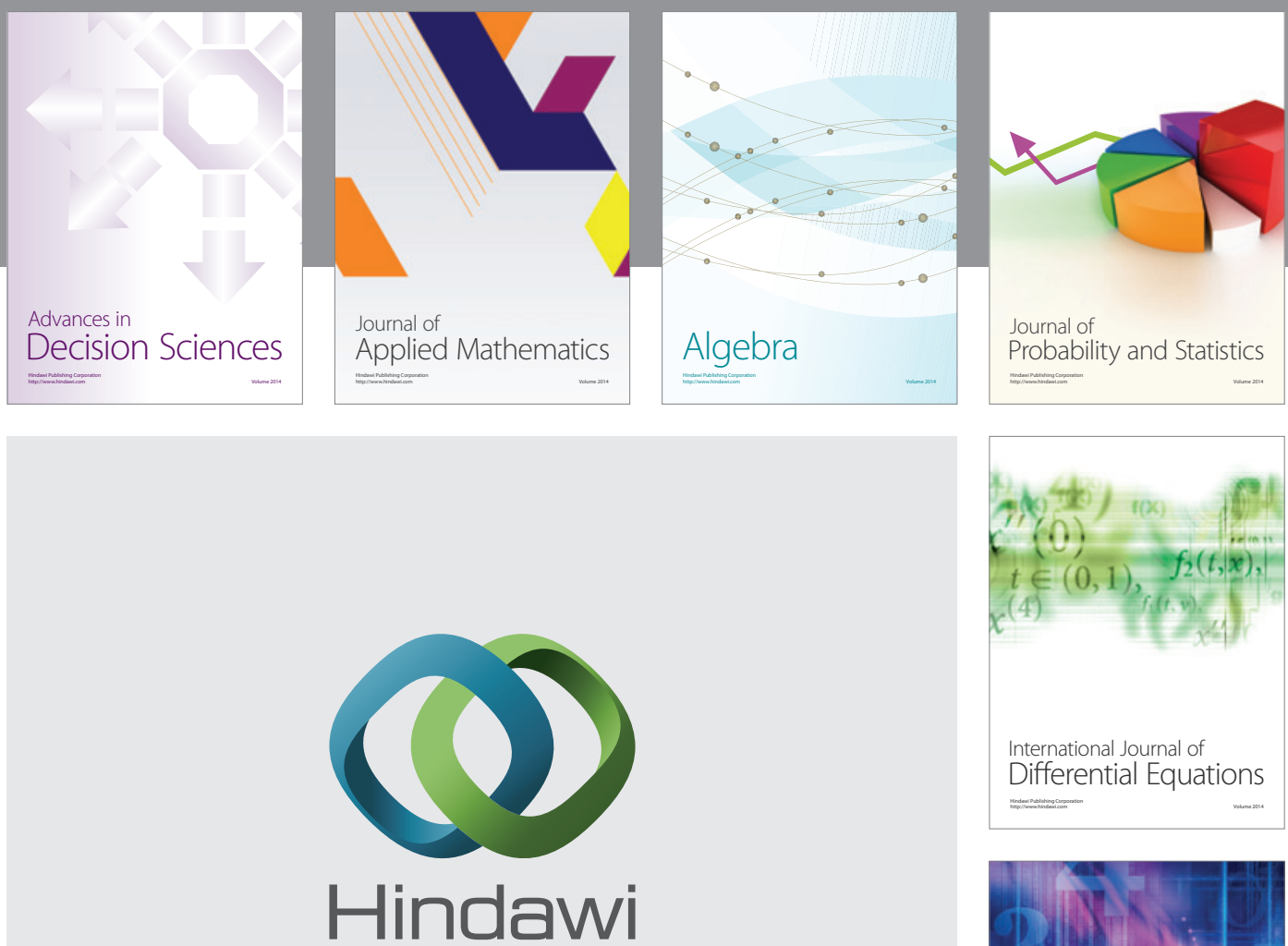

Submit your manuscripts at http://www.hindawi.com
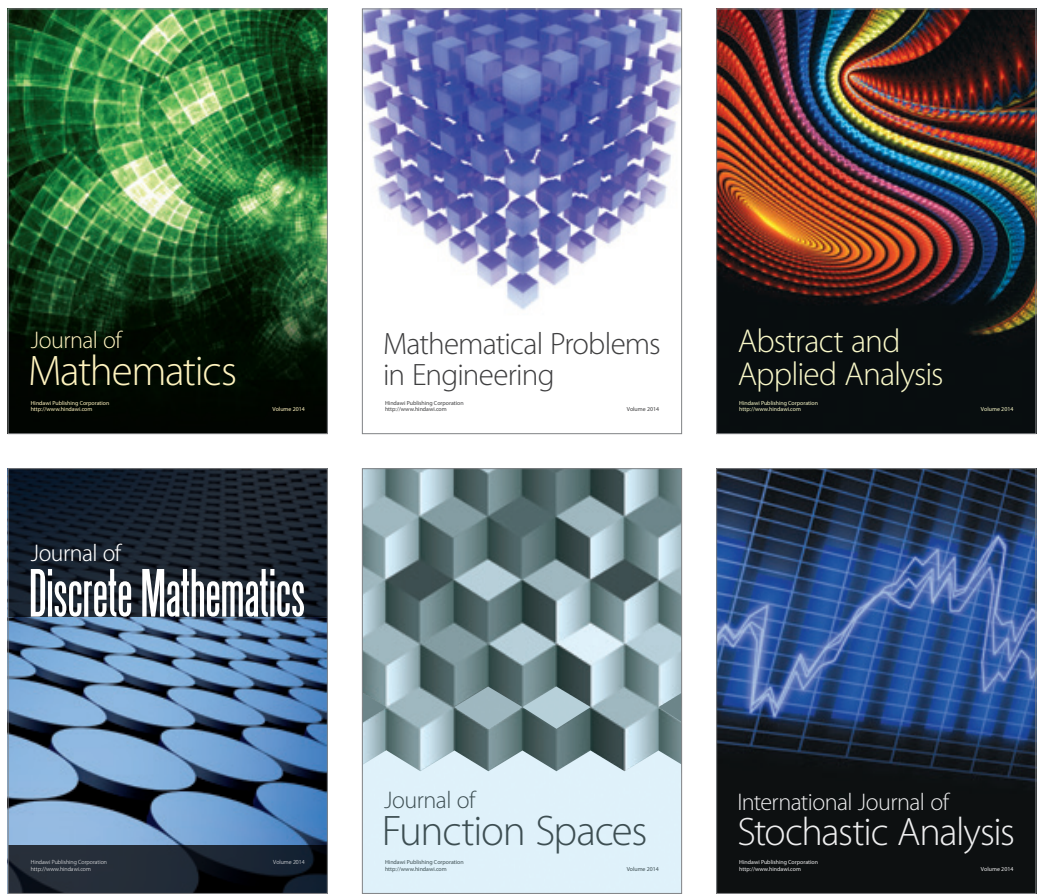

Journal of

Function Spaces

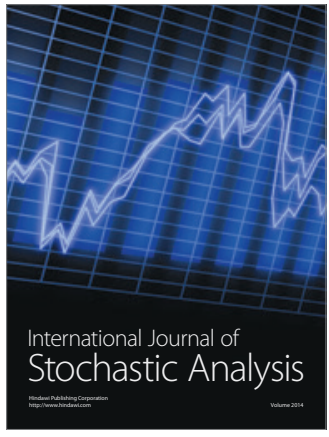

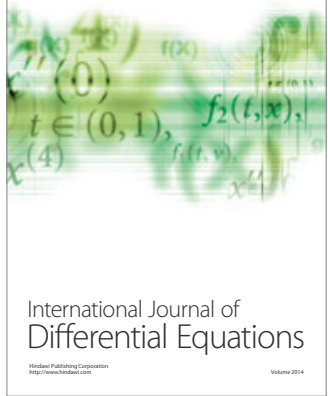
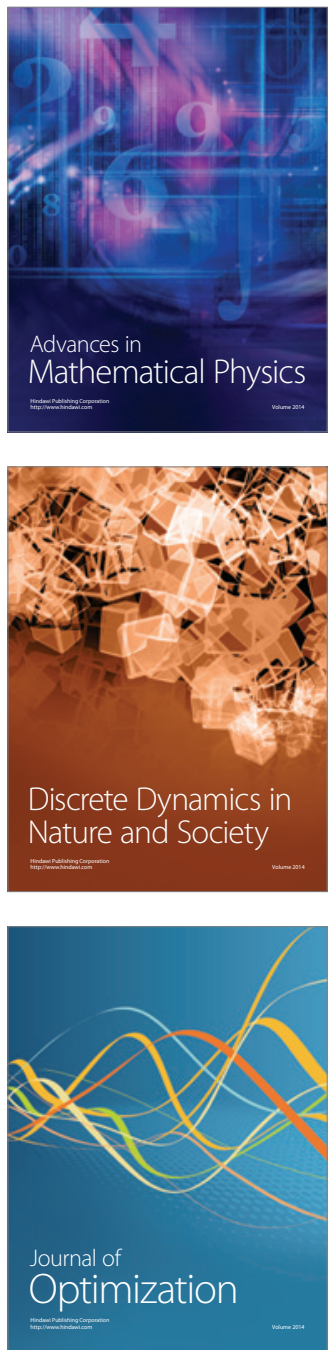